\title{
Metafounder approach for single-step genomic evaluations of Red Dairy cattle
}

\author{
A. A. Kudinov, ${ }^{1,2 *}$ E. A. Mäntysaari, ${ }^{1} \odot$ G. P. Aamand, ${ }^{3} \odot$ P. Uimari, ${ }^{2} \odot$ and I. Strandén ${ }^{1} \odot$ \\ ${ }^{1}$ Natural Resources Institute Finland (Luke), Jokioinen, Finland, FI-31600 \\ ${ }^{2}$ Department of Agricultural Science, University of Helsinki, Helsinki, Finland, FI-00014 \\ ${ }^{3}$ Nordic Cattle Genetic Evaluation, Aarhus, Denmark, DK-8200
}

\section{ABSTRACT}

Single-step genomic BLUP (ssGBLUP) is a powerful approach for breeding value prediction in populations with a limited number of genotyped animals. However, conflicting genomic $(\mathbf{G})$ and pedigree $\left(\mathbf{A}_{22}\right)$ relationship matrices complicate the implementation of ssGBLUP into practice. The metafounder (MF) approach is a recently proposed solution for this problem and has been successfully used on simulated and real multibreed pig data. Advantages of the method are easily seen across breed evaluations, where pedigrees are traced to several pure breeds, which are thereafter used as MF. Application of the MF method to ruminants is complicated due to multi-breed pedigree structures and the inability to transmit existing unknown parent groups (UPG) to MF. In this study, we apply the MF approach for ssGBLUP evaluation of Finnish Red Dairy cattle treated as a single breed. Relationships among MF were accounted for by a (co)variance matrix $(\boldsymbol{\Gamma})$ computed using estimated base population allele frequencies. The attained $\boldsymbol{\Gamma}$ was used to calculate a relationship matrix $\mathbf{A}_{22}^{\Gamma}$ for the genotyped animals. We tested the influence of SNP selection on the $\boldsymbol{\Gamma}$ matrix by applying a minor allele frequency (MAF) threshold $\left(\boldsymbol{\Gamma}_{\mathrm{MAF}}\right)$ where accepted markers had an MAF $\geq 0.05$. Elements in the $\boldsymbol{\Gamma}_{\mathrm{MAF}}$ matrix were slightly lower than in the $\boldsymbol{\Gamma}$ matrix. Correlation between diagonal elements of the genomic and pedigree relationship matrices increased from $0.53\left(\mathbf{A}_{22}\right)$ to $0.76\left(\mathbf{A}_{22}^{\Gamma}\right.$ and $\left.\mathbf{A}_{22}^{\Gamma_{\mathrm{MAF}}}\right)$. Average diagonal elements of $\mathbf{A}_{22}^{\Gamma}$ and $\mathbf{A}_{22}^{\Gamma_{\mathrm{MAF}}}$ matrices increased to the same level as in the $\mathbf{G}$ matrix. The ssGBLUP breeding values (GEBV) were solved using either the original 236 or redefined $8 \mathrm{UPG}$, or $8 \mathrm{MF}$ computed with or without the MAF threshold. For

Received August 21, 2019.

Accepted March 9, 2020.

*Corresponding author: andrei.kudinov@luke.fi bulls, the GEBV validation test results for the 8 UPG and $8 \mathrm{MF}$ gave the same validation reliability $\left(\mathrm{R}^{2} ; 0.31\right)$ and over-dispersion $(0.73$, measured by regression coefficient $b_{1}$ ). No significant $\mathrm{R}^{2}$ increase was observed in cows. Thus, the MF greatly influenced the pedigree relationship matrices but not the GEBV. Selection of SNP according to MAF had a notable effect on the $\boldsymbol{\Gamma}$ matrix and made the $\mathbf{A}_{22}$ and $\mathbf{G}$ matrices more similar. Key words: genetic group, single-step genomic BLUP, metafounder, base population

\section{INTRODUCTION}

Single-step genomic BLUP (ssGBLUP) is an elegant approach for estimating genomic breeding values (GEBV) that uses pedigree $(\mathbf{A})$ and genomic $(\mathbf{G})$ relationship matrices (Aguilar et al., 2010; Christensen and Lund, 2010). The approach has 2 important theoretical assumptions concerning the $\mathbf{A}$ and $\mathbf{G}$ matrices: the same scale and equal base population (Christensen et al., 2012). These assumptions complicate the application of ssGBLUP in dairy cattle breeding. To meet the assumptions, several methods have been proposed that make $\mathbf{G}$ like $\mathbf{A}$. For example, base population allele frequencies (AF) are used (VanRaden, 2008), and elements of $\mathbf{G}$ are scaled and centered to have on average the same diagonal and off-diagonal elements as in A (Vitezica et al., 2011; Christensen et al., 2012). In practice, base population AF are unknown and the $\mathbf{G}$ matrix is often constructed using AF observed in the genotyped population.

Commercial dairy cattle pedigree can seldom be traced to a genetically homogeneous base population because the pedigree often has a complicated breed structure with unknown parent information (VanRaden, 1992; Sponenberg and Bixby, 2007). To solve the problem of incomplete pedigree, Thompson (1979) and Quaas (1988) developed the concept of phantom parents or unknown parent groups (UPG) for animals with unknown parent(s). The UPG are typically assigned according to selection pathways and share the 
same genetics, allowing more accurate estimation of genetic trends in traditional genetic evaluation (Theron et al., 2002). In ssGBLUP, Misztal et al. (2013) observed bias in UPG solutions. The bias increased with an increase in the number of genotyped animals.

The metafounder (MF) approach was proposed by Legarra et al. (2015) to achieve compatibility in the pedigree and genomic relationship matrices. The MF approach combines the idea of using $\mathrm{AF}$ equal to 0.5 for all markers when calculating the $\mathbf{G}$ matrix (Christensen et al., 2012) and assigning unknown parents to MF or pseudo-individuals with self-relationships in the A matrix. The MF are similar to UPG, but allow a related base population with nonzero inbreeding coefficients. The relationships within and between the MF are modeled by a gamma matrix $(\boldsymbol{\Gamma})$, which is used in forming the relationship matrix $\left(\mathbf{A}^{\boldsymbol{\Gamma}}\right)$. The $\boldsymbol{\Gamma}$ matrix may be constructed using an estimated base or observed genotyped population AF (e.g., Legarra et al., 2015; Garcia-Baccino et al., 2017). However, the $\boldsymbol{\Gamma}$ matrix may be poorly estimated when certain AF are estimated inaccurately due to the low number of rare alleles. The large number of UPG increases chances that an UPG is associated with a low number of rare allele genotypes.

Legarra et al. (2015) and Garcia-Baccino et al. (2017) showed the advantage of the MF approach in GEBV estimation using simulated data. Xiang et al. (2017) used the MF method for ssGBLUP evaluation in the crossbreed performance in pigs. According to their results, the MF approach successfully combined 2 breeds in a GEBV evaluation. Pig evaluations clearly focus on the youngest generation and, thus, fewer UPG are needed than in dairy cattle (Arnold et al., 1992). Metafounder approach studies have mostly focused on crossbred and admixture populations (Bradford et al., 2019; van Grevenhof et al., 2019) because the approach may help with implementing ssGBLUP for complicated pedigree populations such as pigs and poultry. However, implementing the MF approach for dairy cattle may be challenging because of the frequently large number of UPG. The few published studies have used simulated dairy cattle data to estimate the $\boldsymbol{\Gamma}$ matrix and its influence on ssGBLUP (Garcia-Baccino et al., 2017; Bradford et al., 2019), but had only a few MF.

We used the MF approach in the ssGBLUP evaluation of 305-d milk production in Finnish Red dairy cattle. We present 2 approaches to estimate the $\boldsymbol{\Gamma}$ matrix, using different numbers of markers. We compared values in the $2 \boldsymbol{\Gamma}$ matrices. The effect of various $\boldsymbol{\Gamma}$ matrices is shown using model validation statistics from ssGBLUP evaluations having either UPG or MF.

\section{MATERIALS AND METHODS}

\section{ssGBLUP Models}

The joint relationship matrix of genotyped and nongenotyped animals in ssGBLUP is commonly denoted as $\mathbf{H}$ (Aguilar et al., 2010; Christensen and Lund, 2010). The $\mathbf{H}^{-1}$ matrix needed in the mixed model equations of ssGBLUP is

$$
\mathbf{H}^{-1}=\mathbf{A}^{-1}+\left(\begin{array}{cc}
0 & 0 \\
0 & \mathbf{G}^{-1}-\mathbf{A}_{22}^{-1}
\end{array}\right),
$$

where $\mathbf{A}$ is the full pedigree relationship matrix, $\mathbf{G}$ is the genomic relationship matrix, and $\mathbf{A}_{22}$ is a pedigree relationship matrix of the genotyped animals.

Single Step with UPG in A. Mean genetic levels of animals with missing parental information were modeled using pedigree-based UPG proposed by Quaas and Pollak (1981). In the UPG model, unknown parents are assumed to be unrelated and completely outbred. The UPG effects in the model only account for possible nonzero expectations in the breeding values of parent groups. There are alternative ways to account for UPG in forming $\mathbf{H}^{-1}$. The standard way is to replace the original $\mathbf{H}^{-1}$ matrix with an augmented one, where the UPG are included as "phantom parents" (Westell et al., 1988). Matilainen et al. (2018), following Misztal et al. (2013), formed the $\mathbf{H}^{-1}$ matrix without groups, and, thereafter, included the UPG via the so-called QP transformation (Quaas and Pollak, 1981) into the final augmented $\mathbf{H}^{-1}$. However, Masuda et al. (2019) recommended omitting the terms involving $\mathbf{G}^{-1}$ in the UPG coefficient part of the augmented $\mathbf{H}^{-1}$ matrix. In our UPG models, the genomic relationship matrix was constructed using VanRaden (2008) method 1 ( $\left.\mathbf{G}_{\mathbf{P v R} 1}\right)$, where base population AF were used to center and scale the marker data. Base population AF were estimated with the GLS model (McPeek et al., 2004) using the Bpop v. 0.30 program (Strandén and Mäntysaari, 2019), which is based on the computational approach described in Strandén et al. (2017). The genomic information was assumed to account for $90 \%$ of the variation in breeding values (i.e., the polygenic proportion was $10 \%$ ). This was attained using a modified $\mathbf{G}$ matrix obtained by averaging original $\mathbf{G}$ and $\mathbf{A}_{22}$ matrices with weights of 0.9 and 0.1 , respectively.

Single Step with Metafounders. In the MF approach, the $\mathbf{H}^{-1}$ matrix was replaced by a modified $\left(\mathbf{H}^{\Gamma}\right)^{-1}$ matrix described by Legarra et al. (2015) and Christensen et al. (2015) as 


$$
\left(\mathbf{H}^{\boldsymbol{\Gamma}}\right)^{-1}=\left(\mathbf{A}^{\boldsymbol{\Gamma}}\right)^{-1}+\left(\begin{array}{cc}
0 & 0 \\
0 & \mathbf{G}_{w}^{-1}-\left(\mathbf{A}_{22}^{\boldsymbol{\Gamma}}\right)^{-1}
\end{array}\right),
$$

where $\mathbf{G}_{w}=(1-w) \mathbf{G}_{05}+w \mathbf{A}_{22}^{\Gamma}, w$ is the proportion of genetic variance not explained by the markers, $\mathbf{G}_{05}=\left(\mathbf{Z}_{101} \mathbf{Z}_{101}^{\prime}\right) \frac{2}{m}, \mathbf{Z}_{101}$ is an $n$ by $m$ marker matrix with genotypes coded by $\{-1,0,1\}, m$ is the number of SNP markers, $n$ is the number of genotyped animals, $\mathbf{A}^{\boldsymbol{\Gamma}}$ is pedigree relationship matrix formed with a $\boldsymbol{\Gamma}$ matrix, and $\mathbf{A}_{22}^{\Gamma}$ is a submatrix of $\mathbf{A}^{\Gamma}$ for the genotyped animals. We used a $10 \%$ polygenic proportion (i.e., $w=$ 0.1 ), as in Garcia-Baccino et al. (2017). The variance covariance structure of the MF can be estimated by $\boldsymbol{\Gamma}$ $=8 \operatorname{Cov}(\mathbf{P})$, as presented in the Appendix of Christensen et al. (2015), where $\mathbf{P}$ was an $m$ by $r$ matrix of $\mathrm{AF}$ and $r$ is the number of MF.

\section{Test Data and Model Validation}

We used Red Dairy cattle (RDC) milk production data provided by Nordic Cattle Genetic Evaluations (NAV). The data sample was extracted from the NAV production evaluation database by including all cows from 426 Finnish herds with at least 10 genotyped cows. This gave 112,479 cows with first-lactation 305-d milk production records produced during 1988-2018. The pedigree included 226,012 animals born in 1960-2016 consisting of $86 \%$ RDC, $12 \%$ Holstein (HOL), $2 \%$ Finn cattle (an indigenous Finnish cattle population), and a total of $1 \%$ of other breeds (Red Holstein, Jersey, Brown Swiss and so on). There were 236 UPG that were based on selection path, birth year, and population of origin. These UPG definitions were the same as those used in the Nordic TD evaluations in November 2018 (Lidauer et al., 2015) and were provided by NAV.

Genotypes were available for 19,757 animals (3,571 bulls and 16,186 cows), which either had observations or were in the pedigree of the animals with observations. Bulls were genotyped using Illumina Bovine SNP50 Bead Chip (Illumina, San Diego, CA) and the cows using a lower-density EuroG 10k chip (http:// www.eurogenomics.com/) that had been imputed to the $50 \mathrm{~K}$ density by NAV. The 29 bovine autosomes available for the analysis had 46,914 markers.

Cow and bull validation data sets were created by removing milk production records for either the last year or for 4 of the previous production years, respectively, as in Gao et al. (2018) and Mäntysaari et al. (2010). We included 101 and 3,551 genotyped test bulls and cows, respectively. Daughter yield deviations (DYD) and yield deviations (YD) were attained using the full data and an animal model by the MiX99 software (Strandén and Lidauer, 1999), as in Gao et al. (2018). The calculated DYD and YD were used for bulls and cows, respectively, in validation regression models (D) $\mathrm{YD}=b_{0}+b_{1} \times$ GEBV, with weights for the DYD observations to estimate general mean $b_{0}$ and regression coefficient $b_{1}$. The weight for DYD was EDC/(EDC + $\lambda)$, where $\lambda$ is $\left(4-h^{2}\right) / h^{2}, h^{2}$ is heritability, and EDC is the bull's effective daughter contributions (https:// interbull.org/ib/cop_appendix4) in evaluation with the full data set. To attain adjusted validation reliability, we divided the model coefficient of determination $\left(\mathrm{R}^{2}\right)$ by the average weight. The regression coefficient $b_{1}$ for the bulls was multiplied by 2 because DYD only represents the sire effect. All the analyses used $h^{2}$ of 0.44 , which is a parameter derived from the NAV milk production test day model for 305-d milk yield. Validation regression analysis was performed in $\mathrm{R}$ software ( $\mathrm{R}$ Development Core Team, 2008).

\section{Unknown Parents and Metafounders}

Eight groups were defined according to the full pedigree structure and replaced the original 236 UPG. We included 6 groups for RDC (birth years <1971, 19711980, 1981-1990, 1991-2000, 2001-2010, 2011-2016), a HOL group, and a group for the other breeds. These 8 groups were treated as UPG or MF. In the MF approach, the base population $\mathrm{AF}$, used to calculate the $\boldsymbol{\Gamma}$ matrix, were estimated using a GLS approach. The GLS model was $\mathbf{m}_{i}=\mathbf{Q} \boldsymbol{\mu}_{i}+\mathbf{e}_{i}$, where $\mathbf{m}_{i}$ is an $n$ by 1 vector of marker $i$ genotypes, $\mathbf{Q}$ is an $n$ by 8 matrix, the rows of which sum up to 1 , and that assigns individuals to fractions of MF, $\boldsymbol{\mu}_{i}$ is an 8 by 1 vector of group means, and $\mathbf{e}_{i} \sim\left(\mathbf{0}, \mathbf{A}_{22}^{*} \sigma^{2}\right)$, where $\mathbf{A}_{22}^{*}$ was the pedigree relationship matrix for the genotyped animals and $\sigma^{2}$ is the common variance. In AF estimation, the common variance need not be known (e.g., Garcia-Baccino et al., 2017). Estimated base population AF for the MF are $\hat{\mathbf{p}}_{i}=(1 / 2) \hat{\boldsymbol{\mu}}_{i}$ for each marker $i=1, \ldots, m$.

To estimate AF for the MF in the GLS model, the $\mathbf{A}_{22}^{*}$ matrix was based on a truncated pedigree, where one parent generation at most was accepted to the genotyped animals. The pedigree truncation guaranteed that the young genotyped animals would contribute to the recent birth year MF and not to the old birth year MF. In addition, the truncation used more genomic information than the full pedigree because genotyped animals had fewer genotyped ancestors but instead a young birth year MF. It can be proven that the GLS method will ignore genotype of an animal whose 
both parents are genotyped and the animal is not an ancestor to a genotyped animal.

The 8 columns of base population AF in the $\mathbf{P}$ matrix were used to estimate the variance covariance structure of the $8 \mathrm{MF}$ or the $\boldsymbol{\Gamma}$ matrix, $\boldsymbol{\Gamma}=8 \operatorname{Cov}(\mathbf{P})$. The effect of minor allele frequencies (MAF) on the MF covariances were tested by creating 2 alternative $\boldsymbol{\Gamma}$ matrices. In the first scenario, the full $\mathbf{P}$ matrix was used to calculate the $\boldsymbol{\Gamma}$ matrix, denoted $\boldsymbol{\Gamma}_{8}$. In the other scenario, denoted $\boldsymbol{\Gamma}_{8 \mathrm{MAF}}$, only those markers with MAF greater or equal to 0.05 in all $\mathrm{RDC}$ cattle $\mathrm{MF}$ were included in the $\mathbf{P}$ matrix. The MAF requirement eliminated 3,783 markers and left 43,131 markers that were used to calculate the $\boldsymbol{\Gamma}_{8 \mathrm{MAF}}$ matrix.

\section{ssGBLUP Computation}

All ssGBLUP calculations used the full pedigree with 226,012 animals and genomic relationship matrices $\left(\mathbf{G}_{\text {PvR } 1}\right.$ or $\left.\mathbf{G}_{05}\right)$ for the 19,757 animals. For the ssGBLUP with MF, the augmented additive relationship matrix of genotyped animals $\left(\mathbf{A}_{22}^{\Gamma}\right)$ was calculated using the modified RelaX2 v. 1.83 program (Strandén and Vuori, 2006). The $\left(\mathbf{G}_{\mathbf{P v R} 1}^{-1}-\mathbf{A}_{22}^{-1}\right)$ and $\left(\mathbf{G}_{w}^{-1}-\left(\mathbf{A}_{22}^{\Gamma}\right)^{-1}\right)$ matrices were calculated using the HGinv v. 0.87 program (Strandén and Mäntysaari, 2018). The latest MiX99 v. 17.1107 (Strandén and Lidauer, 1999) was used to solve the GEBV using the 4 ssGBLUP models. Two of the evaluations were UPG models with either 236 UPG (ssGBLUP 236UPG $_{\text {) }}$ ) or 8 UPG (ssGBLU$\mathbf{P}_{\text {8UPG }}$ ) in $\mathbf{A}$. The UPG were treated as random by adding the inverse of genetic variance to the diagonal of group equations in the mixed model equations. The other 2 ssGBLUP evaluations were MF models that had $8 \mathrm{MF}$, and the pedigree relationship matrices were based on $\boldsymbol{\Gamma}_{8}\left(\operatorname{ssGBLUP}_{\boldsymbol{\Gamma}_{8}}\right)$ or $\boldsymbol{\Gamma}_{8 \mathrm{MAF}}\left(\operatorname{ssGBLUP}_{\boldsymbol{\Gamma}_{8 \mathrm{MAF}}}\right)$. Genetic variance parameters from the model with unrelated founders were used to estimate corresponding parameters for the model with MF. The variance of breeding values in base population descending from MF $\left(\sigma_{a, k}^{2}\right)$ in $\operatorname{ssGBLUP}_{\Gamma_{8}}$ and $\operatorname{ssGBLUP}_{\Gamma_{8 \mathrm{MAF}}}$ models were calculated using the scaling parameter $k$ (i.e., $\sigma_{a, k}^{2}=$ $\left.\sigma_{a}^{2} / k\right)$, where $k=\left[1+\operatorname{tr}(\boldsymbol{\Gamma}) /(2 n)-1^{\prime} \boldsymbol{\Gamma} 1 / n^{2}\right]$ and $\operatorname{tr}(\boldsymbol{\Gamma})$ is the sum of diagonal elements of the $\boldsymbol{\Gamma}$ matrix (Legarra et al., 2015).

\section{Comparisons}

Two traditional ssGBLUP evaluations were computed using different numbers of UPG, and 2 MF-based
ssGBLUP evaluations were computed using different $\boldsymbol{\Gamma}$ matrices and inbreeding coefficients. We present the 2 $\boldsymbol{\Gamma}$ matrices such that the direct effect of the MAF threshold marker selection is seen in elements of the $\boldsymbol{\Gamma}$ matrices. The MF approach is expected to give more similar pedigree and genomic relationship matrices than the traditional pedigree and genomic relationship matrices. In addition, the off-diagonal elements in the pedigree relationship matrix by the MF approach are expected to be higher than in the traditional pedigree relationship matrix. We assessed differences in the diagonal elements (related to the definition of inbreeding) and off-diagonals (related to relatedness) of $\mathbf{A}_{22}^{\Gamma}, \mathbf{A}_{22}$, $\mathbf{G}_{05}$, and $\mathbf{G}_{\text {PvR } 1}$ by correlations and mean differences between these matrices. To identify differences in trends of diagonals to the pedigree and genomic matrices (that are related to breeding selection and changes in inbreeding), average diagonal elements of $\mathbf{A}_{22}^{\Gamma}, \mathbf{G}_{05}$, $\mathbf{G}_{\mathrm{PvR} 1}$, and $\mathbf{A}_{22}$ were plotted by birth year.

The 2 UPG definitions and $2 \mathrm{MF} \boldsymbol{\Gamma}$ matrices gave 4 sets of ssGBLUP predictions. Validation tests used GEBV from the ssGBLUP evaluations separately from the groups of genotyped bulls and cows. Approximately $80 \%$ of bulls born in 1990 to 2014 were genotyped. Thus, differences between the ssGBLUP models may be largest in the genetic trends of the bulls. Averages and standard deviation of selected bull GEBV by birth year were plotted for comparison purposes. The bulls selected for plotting had at least 10 daughters each. Average cow GEBV by birth year were plotted using GEBV from all cows to illustrate the genetic trend in the general population.

\section{RESULTS AND DISCUSSION}

\section{Elements of Matrices}

Table 1 has elements of the $\boldsymbol{\Gamma}_{8}$ and $\boldsymbol{\Gamma}_{8 \mathrm{MAF}}$ matrices. Elements of the $\boldsymbol{\Gamma}_{8 \mathrm{MAF}}$ matrix were slightly lower than corresponding elements in the $\boldsymbol{\Gamma}_{8}$ matrix. All diagonal elements in the $\boldsymbol{\Gamma}$ matrices were less than 1 , which corresponds to negative inbreeding of MF (Table 2) calculated as $F=\gamma-1$, where $\gamma$ is the relationship across gametes (diagonal element of $\boldsymbol{\Gamma}$ ). All elements in the calculated $\boldsymbol{\Gamma}_{8}$ and $\boldsymbol{\Gamma}_{8 \mathrm{MAF}}$ matrices were from 0.452 to 0.797 .

Because the MF were partially formed by breed, the greater than zero off-diagonal elements suggest shared genetics between breeds. Average mean relationship between the RDC and HOL MF was 0.564 and 0.473 in $\boldsymbol{\Gamma}_{8}$ and $\boldsymbol{\Gamma}_{8 \mathrm{MAF}}$, respectively. Off-diagonal elements of the $\boldsymbol{\Gamma}$ matrix between Holstein and Jersey cattle in Legarra et al. (2015) was 0.48 , which is close to the value we 
Table 1. Estimated $\boldsymbol{\Gamma}_{8}$ (lower triangle) and $\boldsymbol{\Gamma}_{8 \mathrm{MAF}}$ (upper triangle) for the metafounders ${ }^{1,2,3}$

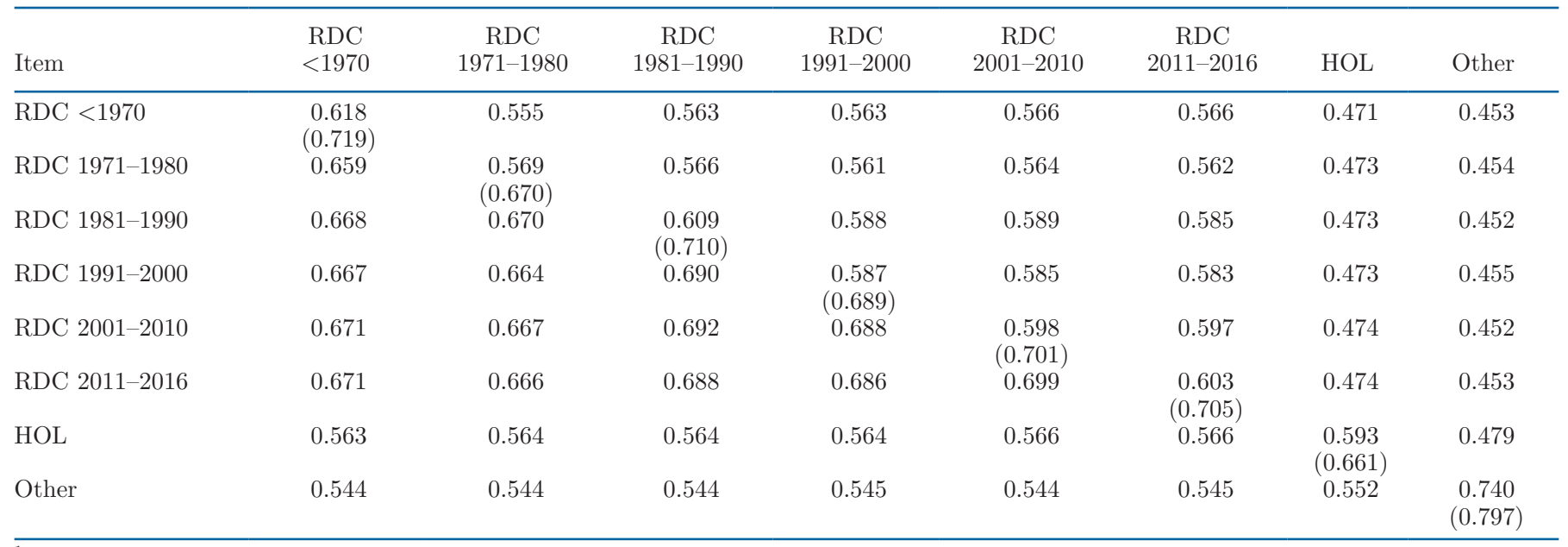

${ }^{1}$ The $\boldsymbol{\Gamma}_{8}$ and $\boldsymbol{\Gamma}_{8 \mathrm{MAF}}$ are covariance matrices between 8 metafounders estimated using all or minor allele frequency $\geq 0.05$ markers, respectively.

${ }^{2}$ The diagonal includes diagonals (i.e., self-relationships of metafounders) of $\boldsymbol{\Gamma}_{8}$ (in parentheses) and $\boldsymbol{\Gamma}_{8 \mathrm{MAF}}$.

${ }^{3}$ Red dairy cattle (RDC) have been divided into metafounders by birth year, Holstein (HOL) cattle have one metafounder, and the other breeds (Other) have been combined into one metafounder.

obtained in $\boldsymbol{\Gamma}_{8 \mathrm{MAF}}$. The authors calculated the $\boldsymbol{\Gamma}$ matrix using published statistics in VanRaden et al. (2011), which included only SNP markers with MAF $\geq 0.05$ (Wiggans et al., 2009). The self-relationships in the HOL and RDC MF in our study were also comparable to 0.55 presented for the HOL and Jersey breeds in Legarra et al. (2015). In our study, an exception to this was the RDC $<1970$ group, which had a diagonal value of 0.618 and 0.719 in $\boldsymbol{\Gamma}_{8 \mathrm{MAF}}$ and $\boldsymbol{\Gamma}_{8}$, respectively. The larger diagonal value in the oldest RDC group may be due to changes in the Finnish RDC breeding program. Before 1970, breeding in the RDC group was mostly limited to Ayrshire cattle with only a low number of imported animals. After 1970, importation began changing the population to more resemble a mixed Nordic RDC breed. Diagonal elements in the group of other breeds were high in both of the $\boldsymbol{\Gamma}$ matrices $(0.740$ and 0.797). This may be due to the influence of Finn Cattle having only a small number of animals, which may produce unreliable AF estimates.

Table 3 shows correlations between (off-)diagonal elements of $\mathbf{A}_{22}, \mathbf{G}_{05}, \mathbf{G}_{\mathrm{PvR} 1}, \mathbf{A}_{22}^{\Gamma_{8}}$, and $\mathbf{A}_{22}^{\Gamma_{8 \mathrm{MAF}}}$ matrices. Constructing $\mathbf{A}_{22}$ using $\boldsymbol{\Gamma}_{8}$ and $\boldsymbol{\Gamma}_{8 \mathrm{MAF}}$ increased the correlation between the diagonal elements of $\mathbf{G}_{05}$ and $\mathbf{A}_{22}$ from 0.66 to 0.76. The diagonal element correlation between elements of $\mathbf{A}_{22}^{\Gamma_{\text {gMAF }}}$ and $\mathbf{A}_{22}$ was higher (0.84) than between $\mathbf{A}_{22}^{\Gamma_{8}}$ and $\mathbf{A}_{22}$ (0.81). The correlation between diagonal elements of $\mathbf{G}_{\mathbf{P v R} 1}$ and $\mathbf{A}_{22}$ decreased from 0.53 to 0.33 and 0.37 for $\mathbf{A}_{22}^{\Gamma_{8 \mathrm{MAF}}}$ and $\mathbf{A}_{22}$, respectively. Despite the high correlation of 0.99 between the diagonal elements of $\mathbf{A}_{22}^{\boldsymbol{\Gamma}_{8}}$ and $\mathbf{A}_{22}^{\boldsymbol{\Gamma}_{8 \mathrm{MAF}}}$, average diagonal elements by the birth year of an animal (Figure 1) were at a higher level for $\mathbf{A}_{22}^{\boldsymbol{\Gamma}_{8}}$ than for $\mathbf{A}_{22}^{\boldsymbol{\Gamma}_{8 \mathrm{MAF}}}$ or $\mathbf{G}_{05}$. Average diagonal elements for both augmented matrices $\left(\mathbf{A}_{22}^{\boldsymbol{\Gamma}_{8}}\right.$ and $\left.\mathbf{A}_{22}^{\boldsymbol{\Gamma}_{8 \mathrm{MAF}}}\right)$ were at the same level as $\mathbf{G}_{05}$ (i.e., from 1.30 to 1.38), whereas the average diagonals of $\mathbf{A}_{22}$ and $\mathbf{G}_{\mathbf{P v R} 1}$ were in range from 0.98 to 1.08 . According to the summary statistics in Table 4, values for the off-diagonal elements of the pedigree relationship matrix $\mathbf{A}_{22}$ increased when using $\boldsymbol{\Gamma}$ to make $\mathbf{A}_{22}^{\boldsymbol{\Gamma}}$. Hence, all elements in the $\mathbf{G}_{05}, \mathbf{A}_{22}^{\boldsymbol{\Gamma}_{8}}$, and $\mathbf{A}_{22}^{\boldsymbol{\Gamma}_{8 \mathrm{MAF}}}$ matrices were higher on average than those in the $\mathbf{A}_{22}$ and $\mathbf{G}_{\mathbf{P v R} 1}$ matrices. Interestingly, both the diagonal and off-diag-

Table 2. Inbreeding coefficients of metafounders calculated using $\boldsymbol{\Gamma}_{8}$ and $\boldsymbol{\Gamma}_{8 \mathrm{MAF}}{ }^{1}$

\begin{tabular}{lcc}
\hline Group $^{2}$ & $\boldsymbol{\Gamma}_{8}$ & $\boldsymbol{\Gamma}_{8 \mathrm{MAF}}$ \\
\hline RDC $<1970$ & -0.28 & -0.38 \\
RDC 1971-1980 & -0.33 & -0.43 \\
RDC 1981-1990 & -0.29 & -0.39 \\
RDC 1991-2000 & -0.31 & -0.41 \\
RDC 2001-2010 & -0.29 & -0.40 \\
RDC 2011-2016 & -0.29 & -0.39 \\
HOL & -0.34 & -0.40 \\
Other & -0.34 & -0.26
\end{tabular}

${ }^{1}$ The $\boldsymbol{\Gamma}_{8}$ and $\boldsymbol{\Gamma}_{8 \mathrm{MAF}}$ are covariance matrices between 8 metafounders estimated using all or minor allele frequency $\geq 0.05$ markers, respectively.

${ }^{2}$ Red dairy cattle (RDC) have been divided into metafounders by birth year, Holstein (HOL) cattle have one metafounder, and the other breeds (Other) have been combined into one metafounder. 
Table 3. Correlation of diagonal (upper triangle) and off-diagonal (lower triangle) elements of $\mathbf{A}_{22}, \mathbf{G}_{05}, \mathbf{G}_{\mathrm{PvR} 1}, \mathbf{A}_{22}^{\boldsymbol{\Gamma}_{8}}$, and $\mathbf{A}_{22}^{\Gamma_{8 \mathrm{MAF}}}$

\begin{tabular}{llllll}
\hline Item $^{1}$ & $\mathbf{A}_{22}$ & $\mathbf{A}_{22}^{\Gamma_{8}}$ & $\mathbf{A}_{22}^{\Gamma_{8 \mathrm{MAF}}}$ & $\mathbf{G}_{05}$ & $\mathbf{G}_{\text {PvR } 1}$ \\
\hline $\mathbf{A}_{22}$ & 1 & 0.81 & 0.84 & 0.66 & 0.53 \\
$\mathbf{A}_{22}^{\Gamma_{8}}$ & 0.89 & 1 & 0.99 & 0.76 & 0.33 \\
$\mathbf{A}_{22}^{\boldsymbol{\Gamma}_{8 \mathrm{MAF}}}$ & 0.92 & 0.99 & 1 & 0.76 & 0.37 \\
$\mathbf{G}_{05}$ & 0.83 & 0.91 & 0.91 & 1 & 0.70 \\
$\mathbf{G}_{\text {PvR } 1}$ & 0.89 & 0.86 & 0.88 & 0.88 & 1 \\
\hline
\end{tabular}

${ }^{1} \mathbf{A}_{22}$ is the pedigree relationship matrix of the genotyped animals; $\mathbf{G}_{05}$ is the genomic relationship matrix with allele frequencies equal to 0.5 $\mathbf{G}_{\mathbf{P v R} 1}$ is the genomic relationship matrix constructed using the VanRaden (2008) method $1 ; \mathbf{A}_{22}^{\Gamma_{8}}$ is the pedigree relationship matrix of genotyped animals augmented by the $\boldsymbol{\Gamma}_{8}$ matrix, and $\mathbf{A}_{22}^{\boldsymbol{\Gamma}_{\text {gMAF }}}$ is the pedigree relationship matrix of genotyped animals augmented by the $\boldsymbol{\Gamma}_{8 \mathrm{MAF}}$ matrix, where the $\boldsymbol{\Gamma}_{8}$ and $\boldsymbol{\Gamma}_{8 \mathrm{MAF}}$ are covariance matrices between 8 metafounders estimated using all or minor allele frequency $\geq 0.05$ markers, respectively.

onal element mean, minimum, and maximum values of $\mathbf{G}_{05}$ and $\mathbf{A}_{22}^{\boldsymbol{\Gamma}_{8 \mathrm{MAF}}}$ agreed very well.

Average inbreeding coefficients in the $\mathbf{A}_{22}$ and $\mathbf{G}_{05}$ matrices were 0.02 and 0.31 , respectively. This difference of 0.29 was close to the 0.272 reported in VanRaden et al. (2011) for HOL cattle (0.056 for $\mathbf{A}_{22}$ and 0.328 for $\left.\mathbf{G}_{05}\right)$. The average inbreeding coefficient increased from 0.02 in $\mathbf{A}_{22}$ to 0.34 and 0.29 in $\mathbf{A}_{22}^{\Gamma_{8}}$ and $\mathbf{A}_{22}^{\Gamma_{8 \mathrm{MAF}}}$, respectively. Following Legarra et al. (2015), a
Table 4. Mean, minimum (Min), and maximum (Max) element values of $\mathbf{A}_{22}, \mathbf{G}_{05}, \mathbf{G}_{\mathrm{PvR} 1}, \mathbf{A}_{22}^{\Gamma_{8}}$, and $\mathbf{A}_{22}^{\Gamma_{\text {gMAF }}}$ from diagonal and off-diagonal ${ }^{1}$

\begin{tabular}{llccc}
\hline Element & Matrix & Mean & Min & Max \\
\hline Diagonal & $\mathbf{A}_{22}$ & 1.02 & 1.00 & 1.29 \\
& $\mathbf{G}_{05}$ & 1.31 & 1.24 & 1.48 \\
& $\mathbf{G}_{\text {PvR1 }}$ & 1.01 & 0.91 & 1.30 \\
& $\mathbf{A}_{22}^{\Gamma_{8}}$ & 1.35 & 1.27 & 1.51 \\
Off-diagonal & $\mathbf{A}_{22}^{\Gamma_{8 \mathrm{MAF}}}$ & 1.31 & 1.23 & 1.50 \\
& $\mathbf{A}_{22}$ & 0.07 & 0.06 & 0.81 \\
& $\mathbf{G}_{05}$ & 0.63 & 0.47 & 1.29 \\
& $\mathbf{G}_{\text {PvR1 }}$ & 0.05 & -0.11 & 0.99 \\
& $\mathbf{A}_{22}^{\boldsymbol{\Gamma}_{8}}$ & 0.72 & 0.54 & 1.22 \\
& $\mathbf{A}_{22}^{\boldsymbol{\Gamma}_{8 \mathrm{MAF}}}$ & 0.62 & 0.45 & 1.16 \\
\hline
\end{tabular}

${ }^{1} \mathbf{A}_{22}$ is the pedigree relationship matrix of the genotyped animals; $\mathbf{G}_{05}$ is the genomic relationship matrix with allele frequencies equal to 0.5 ; $\mathbf{G}_{\mathrm{PvR} 1}$ is the genomic relationship matrix constructed using the VanRaden (2008) method 1; $\mathbf{A}_{22}^{\boldsymbol{\Gamma}_{8}}$ is the pedigree relationship matrix of genotyped animals augmented by the $\boldsymbol{\Gamma}_{8}$ matrix, and $\mathbf{A}_{22}^{\boldsymbol{\Gamma}_{8 \mathrm{MAF}}}$ is the pedigree relationship matrix of genotyped animals augmented by the $\boldsymbol{\Gamma}_{8 \mathrm{MAF}}$ matrix, where the $\boldsymbol{\Gamma}_{8}$ and $\boldsymbol{\Gamma}_{8 \mathrm{MAF}}$ are covariance matrices between 8 metafounders estimated using all or minor allele frequency $\geq 0.05$ markers, respectively.

diagonal element value less than 1 in the $\boldsymbol{\Gamma}$ matrix means a negative individual inbreeding coefficient for MF. In all RDC MF, all elements of $\operatorname{diag}(\boldsymbol{\Gamma})-1$ ranged from -0.38 to -0.43 . We observed the highest self-relationships and corresponding MF inbreeding coefficients in the other breed group, which could be explained by

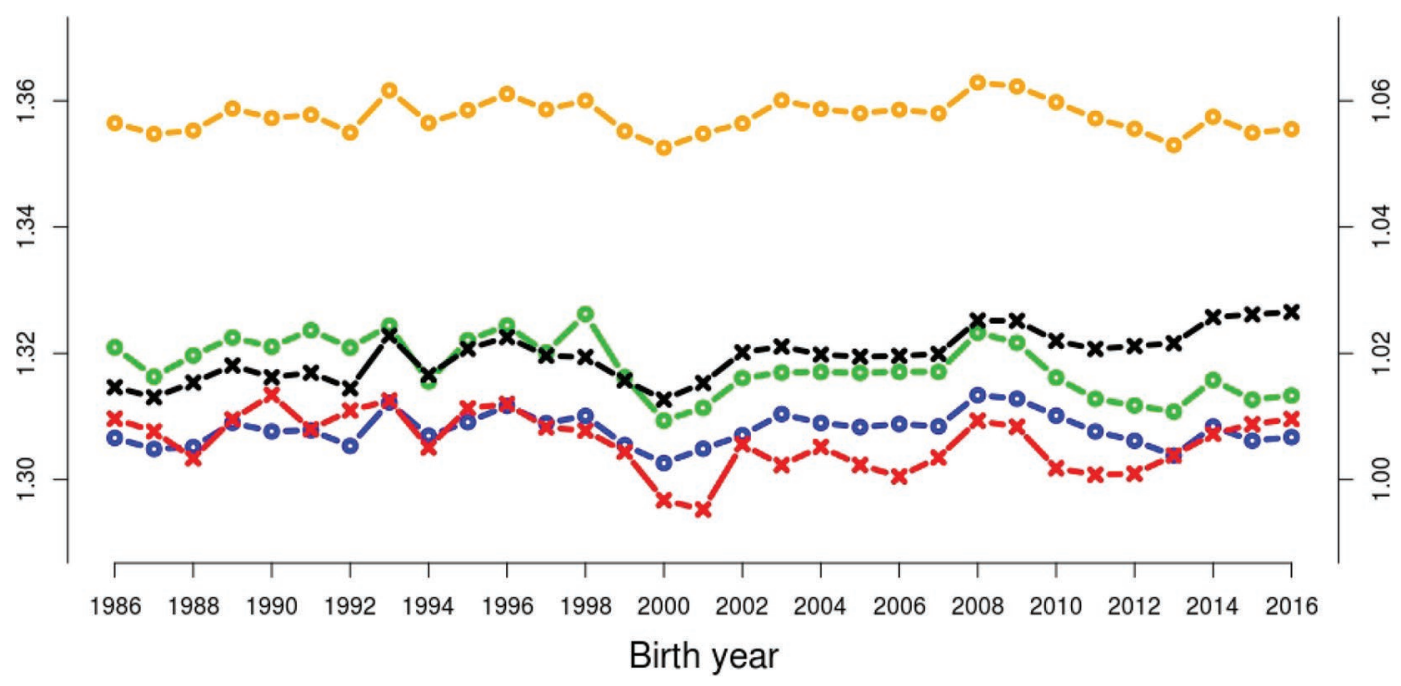

Figure 1. Average diagonal elements of the pedigree relationship matrix of the genotyped animals $\left(\mathbf{A}_{22}\right.$; black cross $)$, the genomic relationship matrix constructed using VanRaden (2008) method 1 ( $\mathbf{G}_{\mathbf{P v R} 1}$; red cross), the genomic relationship matrix assuming that all allele frequencies are equal to $0.5\left(\mathbf{G}_{05}\right.$; green circles), the pedigree relationship matrix of the genotyped animals augmented by $\boldsymbol{\Gamma}_{8}\left(\mathbf{A}_{22}^{\Gamma_{8}} ;\right.$ orange circles), the pedigree relationship matrix of the genotyped animals augmented by $\boldsymbol{\Gamma}_{8 \mathrm{MAF}}\left(\mathbf{A}_{22}^{\boldsymbol{\Gamma}_{8 \mathrm{MAF}}}\right.$; blue circles) by the birth year of an animal. The left side of the y-axis has a scale for $\mathbf{G}_{05}, \mathbf{A}_{22}^{\boldsymbol{\Gamma}_{8}}$, and $\mathbf{A}_{22}^{\boldsymbol{\Gamma}_{8 \mathrm{MAF}}}$, and the right side has a scale for $\mathbf{A}_{22}$ and $\mathbf{G}_{\mathrm{PvR} 1}$. 
the relatively closed small-scale selection program for FinnCattle.

Use of the $\boldsymbol{\Gamma}$ matrix to make the pedigree-based relationship matrix $\mathbf{A}_{22}^{\Gamma_{8}}$ or $\mathbf{A}_{22}^{\Gamma_{8 \mathrm{MAF}}}$ increased the correlation between elements of the pedigree and genomic relationship matrices when compared with the correlation between traditionally formed matrices $\left(\mathbf{G}_{\mathbf{P v R} 1}\right.$ and $\left.\mathbf{A}_{22}\right)$. Correlation between diagonal elements of $\mathbf{A}_{22}^{\Gamma_{8}}$ and $\mathbf{G}_{05}$, as well as between $\mathbf{A}_{22}^{\Gamma_{8 M A F}}$ and $\mathbf{G}_{05}$, was 0.76 , which is higher than the correlation of 0.53 between the diagonal elements of $\mathbf{G}_{\mathrm{PvR} 1}$ and $\mathbf{A}_{22}$. Correlation between the off-diagonal elements of $\mathbf{A}_{22}^{\Gamma_{8}}\left(\mathbf{A}_{22}^{\Gamma_{8 \mathrm{MAF}}}\right)$ and $\mathbf{G}_{05}$ was 0.91 , which is a bit higher than the same correlation (0.89) between $\mathbf{G}_{\mathbf{P v R} 1}$ and $\mathbf{A}_{22}$. Thus, using the $\Gamma$ matrix to form the relationship matrix lifted the diagonal elements of $\mathbf{A}_{22}^{\Gamma}$ matrix to the same level as in the $\mathbf{G}_{05}$ matrix (Figure 1).

The average diagonal of the $\mathbf{A}_{22}^{\Gamma_{8}}$ matrix was at a higher level than the average diagonal of the $\mathbf{G}_{05}$ matrix (Figure 1). Use of the MAF threshold to make $\boldsymbol{\Gamma}_{8 \mathrm{MAF}}$ for $\mathbf{A}_{22}^{\boldsymbol{\Gamma}_{8 \mathrm{MAF}}}$ gave lower average diagonal values than those in $\mathbf{G}_{05}$. In constructing the $\boldsymbol{\Gamma}_{8 \mathrm{MAF}}$ matrix, we deleted the low MAF markers to omit markers with highly uncertain or erroneous AF estimates. This, however, may lead to deleting nearby markers and accepting more markers from certain regions of the genome, particularly if a MAF threshold value higher than 5\% is used. Consequently, AF from various MF may become more similar. For example, 2 breeds may differ due to more intense selection in one of the breeds, leading to the MAF criterion favoring unselected or highly polymorphic markers clustered in certain regions of the genome. Consequently, the $\boldsymbol{\Gamma}$ matrix may show inflated covariances between the MF of these breeds. Linkage disequilibrium (LD) criteria, in which markers are chosen to minimize LD, is an alternative approach to SNP pruning (Hill and Robertson, 1968). Patterns of LD are widely used in marker data quality control and in the analysis of population history for various species (Porto-Neto et al., 2014; Makina et al., 2015; Cañas-Álvarez et al., 2016). Multiple studies have shown persistence in LD levels of various breeds and populations (de Roos et al., 2008; Xu et al., 2019), making LD a potential tool for marker selection.

\section{ssGBLUP Estimation and Validation Results}

The correction factor $k$ used to calculate the variance of breeding values in base population descending from $\operatorname{MF}\left(\sigma_{a, k}^{2}\right)$ in the GEBV calculations for $\operatorname{ssGBLUP}_{\boldsymbol{\Gamma}_{8}}$ and $\operatorname{ssGBLUP}_{\Gamma_{8 \mathrm{MAF}}}$ was 0.72 and 0.77 , respectively. Averages and standard deviations of bull GEBV by birth year are shown in Figures 2 and 3 and the average cow GEBV are shown in Figure 4. We centered the average GEBV trends of cows and bulls, so that the mean GEBV of animals born in 2009 equaled zero. Average bull GEBV in Figure 2 had a similar shape in all the models. The standard deviation (SD) level in Figure 3 for bulls born in 2012-2014 was $20 \mathrm{~kg}$ (3\%) higher in the MF models than in the UPG models. Average cow GEBV by birth year had a similar shape in all models (Figure 4).

Validation test statistics for the approaches are shown in Table 5. Regression coefficients $\left(b_{1}\right)$ were generally slightly higher using MF than UPG. In the bull validation set, we obtained similar adjusted model reliability by $\operatorname{ssGBLUP}_{8 \mathrm{UPG}}, \quad \operatorname{ssGBLUP}_{\Gamma_{8}}, \quad$ and $\operatorname{ssGBLUP}_{\Gamma_{8 \mathrm{MAF}}}$, and the gain was 0.04 in comparison to ssGBLUP $_{236 \mathrm{UPG}}$. In the cow validation set, the validation reliabilities using MF were 0.01 higher than achieved by the UPG models. To exclude pre-selection bias, we conducted the validation tests for bulls also using DYD computed from ssGBLUP ${ }_{236 \mathrm{UPG}}$. The adjusted model reliabilities did not change from those in Table 5 .

Genetic trends in GEBV from the UPG and MF models had a similar shape, showing no effect of the alternative group or founder definitions. We assumed that the inadequate definition of groups would reduce the genetic trend estimate (Tsuruta et al., 2014) but this was not observed. Each of the bulls included in the yearly means in Figures 2 and 3 had at least 10 daughters and, therefore, may be less affected by MF. Perhaps ssGBLUP predictions where most of the sires are genotyped are robust against the definition of UPG or MF. Meyer et al. (2018) reported a slightly higher estimated genetic trend with the MF approach compared with ssGBLUP without groups. However, females were the most often genotyped group in their data. Also, the SD of the GEBV were fairly similar between all evaluations (Figure 3). The unstandardized genetic levels in the MF models were at a higher level compared with the UPG models. This difference did not affect the animal rankings by GEBV but indicate that the models defined base populations differently. We observed a high correlation of bull GEBV between the MF model and the original 236 UPG model (0.972), whereas the correlation of GEBV between the MF model and the 8 UPG model was much lower (0.931; correlations not given in tables).

We used pedigree-based UPG in the ssGBLUP model via incomplete QP transformation (Quaas and Pollak, 
1981), that is, QP transformation for $\mathbf{A}^{-1}$ instead of $\mathbf{H}^{-1}$. In case of a multi-breed structure [i.e., for the joint Nordic (Denmark, Finland, Sweden) RDC genetic evaluation], Matilainen et al. (2018) proposed to use QP transformation in $\mathbf{H}^{-1}$ (Misztal et al., 2013). Bradford et al. (2019) observed that the incomplete QP transformation in ssGBLUP may be applied successfully by accounting for $\mathbf{A}^{-1}$ only, when a purebred population is analyzed. The MF approach used in this study could be a smooth way to implement the ssGBLUP model for the joint Nordic evaluation.

\section{Estimation of AF}

Defining the base population is the greatest challenge in the MF approach. We focused on 2 issues: the number of MF and the genetic change in time. Simply replacing current UPG by MF is often impossible in genetic evaluations of large commercial populations, which have many UPG and animals with missing parents. We combined all UPG by breed and split the RDC-based UPG by decade to form $8 \mathrm{MF}$. For the HOL and "other" breeds, the limited number of animals and absence of phenotypic data were the key reasons for using only one MF per breed. By using multiple MF in RDC, we could account for a possible change in $\mathrm{AF}$ with time.

Base population AF for the MF are needed to calculate the $\boldsymbol{\Gamma}$ matrix. Garcia-Baccino et al. (2017) presented 3 approaches for estimating base population AF to be used for populations with crossbreed animals. All of these methods use genotypes and a pedigree relationship matrix or matrices. We used the genetics group model utilizing GLS. An alternative GLS approach allows differences between gene content variances across breeds and relies on a multi-breed model presented in García-Cortés and Toro (2006). All the pedigree-based approaches only need the pedigree of ancestors to the genotyped animals, and the base population groups are defined by MF through pedigree information. However, the unbalanced distribution of genotyped animals to UPG or MF in the full pedigree affects all base population AF estimation methods that rely on the pedigree relationship matrix.

In our study, a major part of the genotyped animals (75\%) contributed to the oldest RDC group (RDC $<1971$ ) when the full pedigree was used, although most of the genotyped animals (90.6\%) were born after 2000 . Thus, the contribution gained from genotypes of animals born in 2000-2016 to the recent year groups would be small and would depend on pedigree incompleteness. Consequently, the base population AF of the oldest RDC groups would be well estimated with, possibly, a small influence from young animal genotypes. To solve these issues in the base population AF estimation for the MF, we limited the length of the pedigree of genotyped animals by only accepting ungenotyped animals with genotyped offspring.

In our study, we calculated the base population AF of HOL and the other breeds group using the ancestor

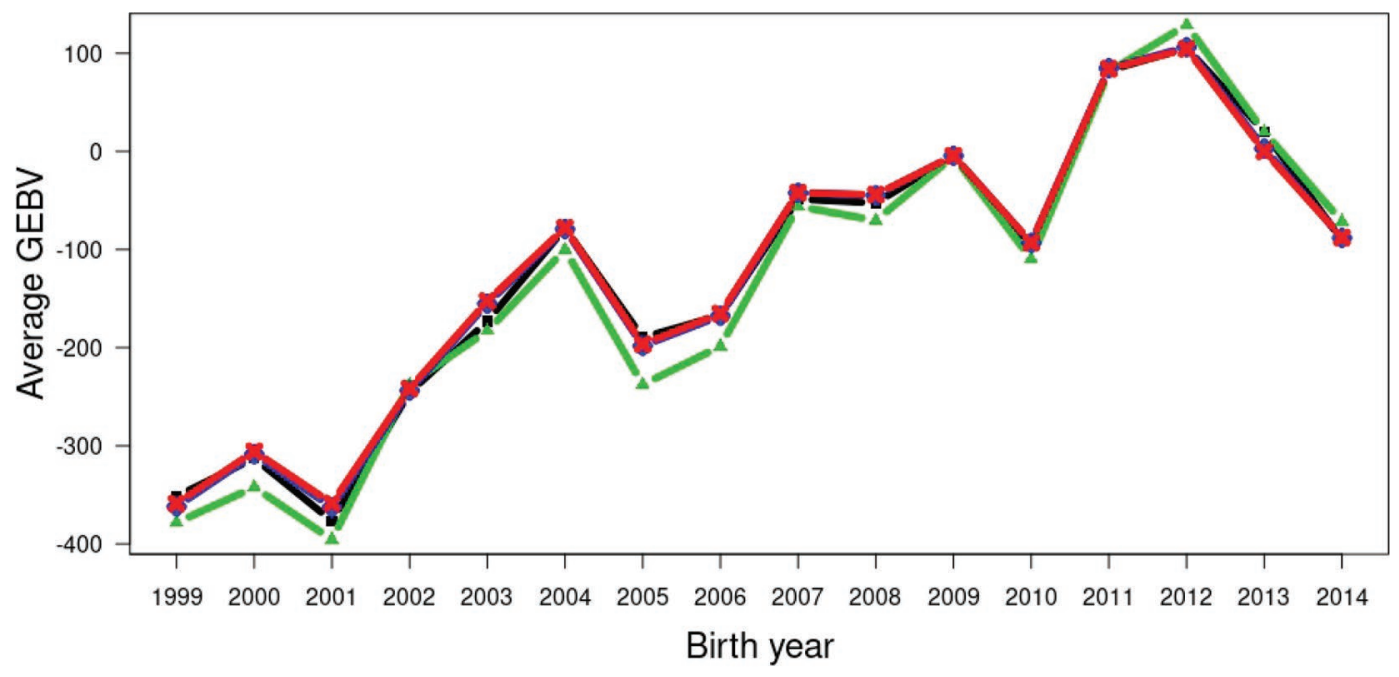

Figure 2. Average genomic breeding value (GEBV) of bulls by birth year in 305-d milk yield (kg). Each bull had at least 10 daughters. The lines are from the unknown parent group (UPG) models ssGBLUP ${ }_{236 \mathrm{UPG}}$ (single-step genomic BLUP for 236 UPG; black square) and ssGBLU$\mathrm{P}_{8 \mathrm{UPG}}$ (single-step genomic BLUP for $8 \mathrm{UPG}$; green triangle) and from the metafounder models ssGBLUP ${ }_{\Gamma_{8}}$ (single-step genomic BLUP with covariance matrices between 8 metafounders; blue diamond) and $\operatorname{ssGBLUP}_{\Gamma_{8 \mathrm{MAF}}}$ (single-step genomic BLUP with covariance matrices between 8 metafounders estimated using minor allele frequency $\geq 0.05$; red cross). 
structure of genotyped RDC animals only. We tested the applicability of the chosen GLS approach by estimating an additional $\boldsymbol{\Gamma}$ matrix $\left(\boldsymbol{\Gamma}_{\mathrm{RDC} \& H O L}\right.$, Table 6$)$. The matrix was calculated using HOL AF (M. Koivula, Natural Resources Institute Finland, Jokoinen, 2019, personal communication). We estimated these AF with HOL breed genotypes and the pedigree used in Koivula et al. (2018). The estimated $\boldsymbol{\Gamma}_{\mathrm{RDC} \& H O L}$ was compared with the presented $\boldsymbol{\Gamma}_{8}$ and $\boldsymbol{\Gamma}_{8 \mathrm{MAF}}$ matrices (Table 1), which were only based on genotyped RDC animals. The closeness of the average diagonal values in the HOL MF of $\boldsymbol{\Gamma}_{\mathrm{RDC} \& \mathrm{HOL}}(0.615), \boldsymbol{\Gamma}_{8}(0.661)$, and $\boldsymbol{\Gamma}_{8 \mathrm{MAF}}(0.593)$ suggest that we were able to estimate the $\boldsymbol{\Gamma}$ matrices fairly well without including the pure HOL population genotypes. In addition, the MAF-based marker selection gave the closest value to the HOL genotypes-derived value. Using the truncated pedigree is one possible reason for the good estimation of HOL AF using RDC data. The aim of the pedigree truncation was to distribute available genotypes evenly across MF. Pruning the pedigree appeared to solve 2 important problems: unequal distribution of genotyped animals across MF and the mixture of AF breed groups.

Off-diagonal elements of the $\boldsymbol{\Gamma}$ matrix suggested fairly high similarity between all founder groups. We tested a $\boldsymbol{\Gamma}$ matrix where the off-diagonal elements were half of those in the estimated $\boldsymbol{\Gamma}$ matrix (results not shown). This half-reduced off-diagonal element $\boldsymbol{\Gamma}$ matrix nearly gave the same GEBV solutions, with a correlation of 0.998. Thus, for this data set, the MF-based ssGBLUP evaluation does not seem to be very sensitive to the off-diagonal element values in the $\boldsymbol{\Gamma}$ matrix. Further work is needed to ascertain that this can be generalized to data sets with more genotyped animals and different population structure.

We observed differences in the $\boldsymbol{\Gamma}$ matrix depending on the set of markers used to estimate the $\boldsymbol{\Gamma}$ matrix. When markers were required to have an MAF above a certain limit, values in the $\boldsymbol{\Gamma}$ matrix were lower than when all the markers were used. This is to be expected because the $\boldsymbol{\Gamma}$ matrix is estimated by the variance of $\mathrm{AF}$ and the MAF threshold reduced range of marker $\mathrm{AF}$ is used to calculate the variance. The case is similar to that in Chen et al. (2011) where increasing the MAF threshold in the marker selection decreased the values of (off-)diagonal elements in the genomic relationship matrix. The $\boldsymbol{\Gamma}$ matrix is a function of the chosen MAF threshold as a consequence of the marker selection. We must therefore be careful when making interpretations of values in the estimated $\boldsymbol{\Gamma}$ matrix. For example, the MAF threshold was applied to all of the RDC-based MF, but the set of selected markers will change if the HOL animals have genotypes.

The pedigree pruning approach allowed estimation of base population $\mathrm{AF}$ for the breed groups despite all the genotyped animals being from the RDC breeding program. Still, it is impossible to model AF changes in base populations and MF before the first genotyped parent generations. One possibility is to assume that the AF changes have continuity and that the changes can also be extrapolated to early years before the genotyping began. Then the variance structures of $\boldsymbol{\Gamma}$ in the

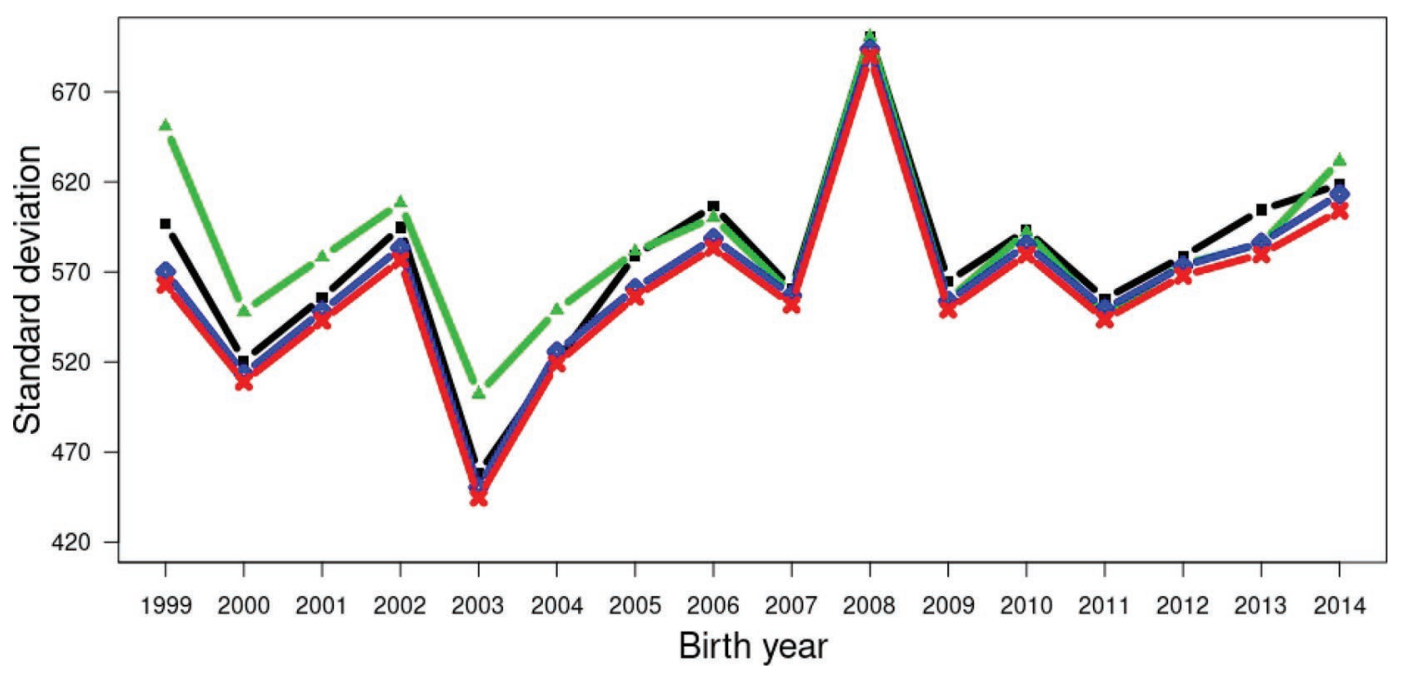

Figure 3. Standard deviation of bull genomic breeding values by birth year in 305-d milk yield (kg). Each bull had at least 10 daughters. Trends are from the unknown parent group (UPG) models ssGBLUP ${ }_{236 \mathrm{UPG}}$ (single-step genomic BLUP for 236 UPG; black square) and ssGBLUP $_{8 U P G}$ (single-step genomic BLUP for 8 UPG; green triangle) and from the metafounder models ssGBLUP $\Gamma_{8}$ (single-step genomic BLUP with covariance matrices between 8 metafounders; blue diamond) and $\operatorname{ssGBLUP}_{\Gamma_{8 \mathrm{MAF}}}$ (single-step genomic BLUP with covariance matrices between 8 metafounders estimated using minor allele frequency $\geq 0.05$; red cross). 
Table 5. Genomic estimated breeding value (GEBV) validation test regression coefficients and validation reliabilities of single-step genomic BLUP (ssGBLUP) GEBV for genotyped bulls and cows ${ }^{1,2}$

\begin{tabular}{|c|c|c|c|c|c|c|c|}
\hline $\begin{array}{l}\text { Validation } \\
\text { set }\end{array}$ & Model $^{3}$ & $b_{0}$ & $\mathrm{SE}$ & $b_{1}$ & $\mathrm{SE}$ & $\mathrm{R}^{2}$ & $\mathrm{R}_{\mathrm{EDC}}^{2}$ \\
\hline \multirow[t]{3}{*}{ Bulls } & $\begin{array}{l}\text { ssGBLUP }_{236 U P G} \\
\text { ssGBLUP }_{\text {SLPG }}\end{array}$ & $\begin{array}{l}70 \\
18\end{array}$ & $\begin{array}{l}16 \\
16\end{array}$ & $\begin{array}{l}0.61 \\
0.73\end{array}$ & $\begin{array}{l}0.06 \\
0.06\end{array}$ & 0.23 & 0.27 \\
\hline & $\operatorname{ssGBLUP}_{\Gamma_{8}}$ & -22 & 22 & 0.72 & 0.06 & 0.26 & 0.31 \\
\hline & $\operatorname{ssGBLUP}_{\Gamma_{8 \mathrm{MAF}}}$ & -27 & 23 & 0.73 & 0.06 & 0.26 & 0.31 \\
\hline \multirow[t]{4}{*}{ Cows } & ssGBLUP $_{236 \mathrm{UPG}}$ & 118 & 9 & 0.89 & 0.03 & 0.16 & 0.36 \\
\hline & ssGBLUP $_{8 \mathrm{UPG}}$ & 150 & 8 & 0.89 & 0.03 & 0.16 & 0.36 \\
\hline & $\operatorname{ssGBLUP}_{\Gamma_{8}}$ & 12 & 13 & 0.90 & 0.03 & 0.16 & 0.37 \\
\hline & $\operatorname{ssGBLUP}_{\Gamma_{8 \mathrm{MAF}}}$ & -0.2 & 13 & 0.93 & 0.04 & 0.16 & 0.37 \\
\hline
\end{tabular}

${ }^{1}$ Regression coefficient $b_{1}$ in the equation daughter yield deviations (DYD) $=b_{0}+b_{1} \times$ GEBV for the bulls has been multiplied by 2 unlike the general mean $b_{0}$.

${ }^{2} R^{2}$ is the coefficient of determination from the validation regression; $R_{E D C}^{2}$ is adjusted by the average reliability of phenotypes in the validation group.

${ }^{3}$ Model ssGBLUP $_{236 \mathrm{UPG}}\left(\mathrm{ssGBLUP}_{8 \mathrm{UPG}}\right.$ ) had 236 (8) unknown parent groups; ssGBLUP ${ }_{\Gamma_{8}}$ had 8 metafounders with the metafounder $\boldsymbol{\Gamma}$ matrix calculated using all markers; ssGBLUP $_{\Gamma_{8 \mathrm{MAF}}}$ used markers with a minor allele frequency $\geq 0.05$ in the metafounder $\boldsymbol{\Gamma}$ matrix calculation.

observed base populations (i.e., parents of genotyped animals) could be extended to describe variances of unobservable MF using covariance functions (Kirkpatrick et al., 1994) with appropriate breeds and birth years.

\section{CONCLUSIONS}

We tested the MF approach on RDC data with a complicated multi-breed structure. The original 236 UPG were replaced by $8 \mathrm{MF}$ and tested in ssGBLUP evaluation. Use of MF increased correlation between elements of the pedigree and genomic relationship matrices. Introduction of MAF-based marker selection before computing the $\boldsymbol{\Gamma}$ matrix for the MF gave $\mathbf{A}_{22}^{\Gamma_{\text {gMAF }}}$ an advantage over the original $\mathbf{A}_{22}^{\Gamma_{8}}$ in correlations with elements of the genomic relationship matrix. The reduction of UPG groups from 236 to 8 reduced the inflation in the predictions and increased validation accuracy. The GEBV from models with $8 \mathrm{MF}$ gave almost

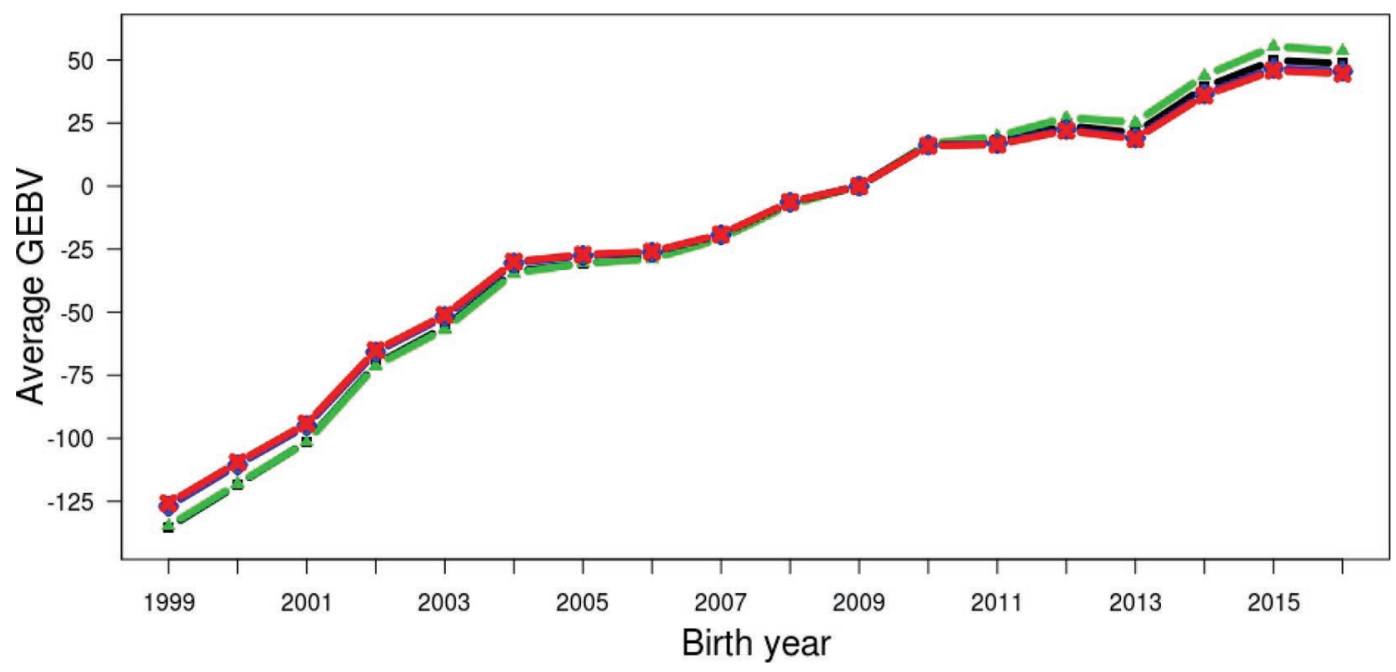

Figure 4. Average genomic breeding value (GEBV) of cows by birth year in 305-d milk yield (kg). The lines are from the unknown parent group (UPG) models ssGBLUP ${ }_{236 \mathrm{UPG}}$ (single-step genomic BLUP for 236 UPG; black square) and ssGBLUP 8UPG $_{\text {(single-step genomic BLUP for }}$ 8 UPG; green triangle) and from the metafounder models ssGBLUP $_{\Gamma_{8}}$ (single-step genomic BLUP with covariance matrices between 8 metafounders; blue diamond) and $\operatorname{ssGBLUP}_{\Gamma_{8 \mathrm{MAF}}}$ (single-step genomic BLUP with covariance matrices between 8 metafounders estimated using minor allele frequency $\geq 0.05$; red cross). 


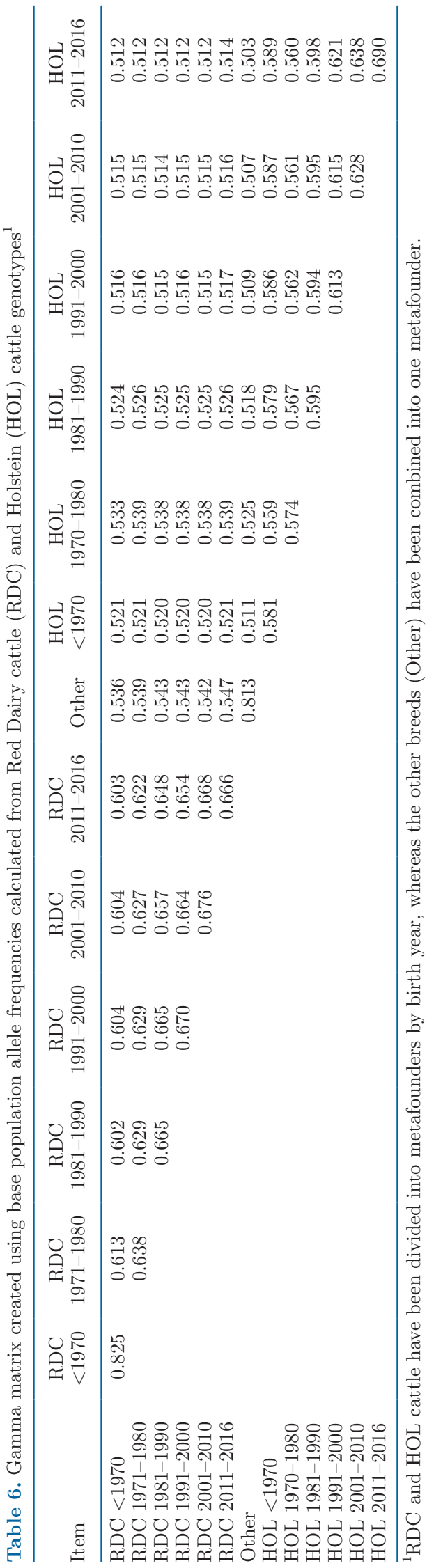

the same validation results and genetic trends as the 8 UPG. Future development should focus on ways to increase the number of MF closer to the number of UPG.

\section{ACKNOWLEDGMENTS}

We acknowledge Viking Genetics (Randers, Denmark) and Nordic Cattle Genetic Evaluation (Aarhus, Denmark) for providing the genotype data and participating in project financing. The authors have not stated any conflicts of interest.

\section{REFERENCES}

Aguilar, I., I. Misztal, D. L. Johnson, A. Legarra, S. Tsuruta, and T. J. Lawlor. 2010. Hot topic: A unified approach to utilize phenotypic, full pedigree, and genomic information for genetic evaluation of Holstein final score. J. Dairy Sci. 93:743-752. https://doi.org/10 $.3168 /$ jds.2009-2730.

Arnold, J. W., J. K. Bertrand, and L. L. Benyshek. 1992. Animal model for genetic evaluation of multi-breed data. J. Anim. Sci. 70:3322-3332. https://doi.org/10.2527/1992.70113322x.

Bradford, H. L., Y. Masuda, P. M. VanRaden, A. Legarra, and I. Misztal. 2019. Modeling missing pedigree in single-step genomic BLUP. J. Dairy Sci. 102:2336-2346. https://doi.org/10.3168/jds .2018-15434.

Cañas-Álvarez, J. J., E. F. Mouresan, L. Varona, C. Díaz, A. Molina, J. A. Baro, J. Altarriba, M. J. Carabaño, J. Casellas, and J. Piedrafita. 2016. Linkage disequilibrium, persistence of phase, and effective population size in Spanish local beef cattle breeds assessed through a high-density single nucleotide polymorphism chip. J. Anim. Sci. 94:2779-2788. https://doi.org/10.2527/jas.2016-0425.

Chen, C. Y., I. Misztal, I. Aguilar, A. Legarra, and W. M. Muir. 2011. Effect of different genomic relationship matrices on accuracy and scale. J. Anim. Sci. 89:2673-2679. https://doi.org/10.2527/ jas.2010-3555.

Christensen, O. F., A. Legarra, M. S. Lund, and G. Su. 2015. Genetic evaluation for three-way crossbreeding. Genet. Sel. Evol. 47:98. https://doi.org/10.1186/s12711-015-0177-6.

Christensen, O. F., and M. S. Lund. 2010. Genomic prediction when some animals are not genotyped. Genet. Sel. Evol. 42:2. https:// doi.org/10.1186/1297-9686-42-2.

Christensen, O. F., P. Madsen, B. Nielsen, T. Ostersen, and G. Su. 2012. Single-step methods for genomic evaluation in pigs. Animal 6:1565-1571. https://doi.org/10.1017/S1751731112000742.

de Roos, A. P., B. J. Hayes, R. J. Spelman, and M. E. Goddard 2008. Linkage disequilibrium and persistence of phase in HolsteinFriesian, Jersey and Angus cattle. Genetics 179:1503-1512. https: //doi.org/10.1534/genetics.107.084301.

Gao, H., M. Koivula, J. Jensen, I. Strandén, P. Madsen, T. Pitkänen, G. P. Aamand, and E. A. Mäntysaari. 2018. Short communication: Genomic prediction using different single-step methods in the Finnish red dairy cattle population. J. Dairy Sci. 101:10082-10088. https://doi.org/10.3168/jds.2018-14913.

Garcia-Baccino, C. A., A. Legarra, O. F. Christensen, I. Misztal, I Pocrnic, Z. G. Vitezica, and R. J. Cantet. 2017. Metafounders are related to Fst fixation indices and reduce bias in single-step genomic evaluations. Genet. Sel. Evol. 49:34. https://doi.org/10 $.1186 / \mathrm{s} 12711-017-0309-2$.

García-Cortés, L. A., and M. A. Toro. 2006. Multibreed analysis by splitting the breeding values. Genet. Sel. Evol. 38:601-615. https:/ /doi.org/10.1051/gse:2006024.

Hill, W. G., and A. Robertson. 1968. Linkage disequilibrium in finite populations. Theor. Appl. Genet. 38:226-231. https://doi.org/10 .1007/BF01245622.

Kirkpatrick, M., W. G. Hill, and R. Thompson. 1994. Estimating the covariance structure of traits during growth and ageing, illustrated 
with lactation in dairy cattle. Genet. Res. 64:57-69. https://doi org/10.1017/S0016672300032559.

Koivula, M., I. Strandén, G. P. Aamand, and E. A. Mäntysaari. 2018. Comparison of ssGBLUP and ssGTBLUP using Nordic Holstein TD data. Proc. World Congress on Genetics Applied to Livestock Production. 11:445.

Legarra, A., O. F. Christensen, Z. G. Vitezica, I. Aguilar, and I. Misztal. 2015. Ancestral relationships using metafounders: Finite ancestral populations and across population relationships. Genetics 200:455-468. https://doi.org/10.1534/genetics.115.177014.

Lidauer, M. H., J. Pösö, J. Pedersen, J. Lassen, P. Madsen, E. A. Mäntysaari, U. S. Nielsen, J.-Á. Eriksson, K. Johansson, T. Pitkänen, I. Strandén, and G. P. Aamand. 2015. Across-country test-day model evaluations for Holstein, Nordic Red Cattle, and Jersey. J. Dairy Sci. 98:1296-1309. https://doi.org/10.3168/jds.2014-8307.

Makina, S. O., J. F. Taylor, E. Van Marle-Köster, F. C. Muchadeyi, M. L. Makgahlela, M. D. MacNeil, and A. Maiwashe. 2015. Extent of linkage disequilibrium and effective population size in four South African Sanga cattle breeds. Front. Genet. 6:337. https:// doi.org/10.3389/fgene.2015.00337.

Mäntysaari, E. A., Z. Liu, and P. VanRaden. 2010. Interbull validation test for genomic evaluations. Interbull Bull. 41:17-22.

Masuda, Y., S. Tsuruta, E. Nicolazzi, and I. Misztal. 2019. Singlestep GBLUP including more than 2 million genotypes with missing pedigrees for production traits in US Holstein. Interbull Open Meeting, Cincinnati, OH. https://interbull.org/static/web/10_30 _Masuda_final.pdf.

Matilainen, K., I. Strandén, G. P. Aamand, and E. A. Mäntysaari. 2018. Single step genomic evaluation for female fertility in Nordic Red dairy cattle. J. Anim. Breed. Genet. 135:337-348. https://doi .org/10.1111/jbg. 12353.

McPeek, M. S., X. Wu, and C. Ober. 2004. Best linear unbiased allelefrequency estimation in complex pedigrees. Biometrics 60:359-367. https://doi.org/10.1111/j.0006-341X.2004.00180.x.

Meyer, K., B. Tier, and A. Swan. 2018. Estimates of genetic trend for single-step genomic evaluations. Genet. Sel. Evol. 50:39. https:// doi.org/10.1186/s12711-018-0410-1.

Misztal, I., Z. G. Vitezica, A. Legarra, I. Aguilar, and A. A. Swan. 2013. Unknown-parent groups in single-step genomic evaluation. J. Anim. Breed. Genet. 130:252-258. https://doi.org/10.1111/jbg .12025

Porto-Neto, L. R., J. W. Kijas, and A. Reverter. 2014. The extent of linkage disequilibrium in beef cattle breeds using high-density SNP genotypes. Genet. Sel. Evol. 46:22. https://doi.org/10.1186/ 1297-9686-46-22.

Quaas, R. L. 1988. Additive genetic model with groups and relationships. J. Dairy Sci. 71:1338-1345. https://doi.org/10.3168/jds .S0022-0302(88)79691-5.

Quaas, R. L., and E. J. Pollak. 1981. Modified equations for sire models with groups. J. Dairy Sci. 64:1868-1872. https://doi.org/10 .3168/jds.S0022-0302(81)82778-6.

R Development Core Team. 2008. R: A language and environment for statistical computing. R Foundation for Statistical Computing, Vienna, Austria. http://www.R-project.org.

Sponenberg, D. P., and D. E. Bixby. 2007. Managing Breeds for a Secure Future: Strategies for Breeders and Breed Associations. American Livestock Breeds Conservancy, Pittsboro, NC.

Strandén, I., and M. Lidauer. 1999. Solving large mixed models using preconditioned conjugate gradient iteration. J. Dairy Sci. 82:27792787. https://doi.org/10.3168/jds.S0022-0302(99)75535-9.

Strandén, I., and E. A. Mäntysaari. 2018. HGinv Program. Natural Resources Institute Finland (LUKE).

Strandén, I., and E. A. Mäntysaari. 2019. Bpop Program. Natural Resources Institute Finland (LUKE).
Strandén, I., K. Matilainen, G. P. Aamand, and E. A. Mäntysaari. 2017. Solving efficiently large single-step genomic best linear unbiased prediction models. J. Anim. Breed. Genet. 134:264-274. https://doi.org/10.1111/jbg.12257.

Strandén, I., and K. Vuori. 2006. RelaX2: Pedigree analysis program. Pages 27-30 in Proc. 8th World Congr. Genet. Appl. Livest. Prod., Belo Horizonte, MG, Brazil. http://www.wcgalp.org/proceedings/ 2006/relax2-pedigree-analysis-programme.

Theron, H. E., F. H. J. Kanfer, and L. Rautenbach. 2002. The effect of phantom parent groups on genetic trend estimation. S. Afr. J. Anim. Sci. 32:130-135. https://doi.org/10.4314/sajas.v32i2.3755.

Thompson, R. 1979. The estimation of heritability with unbalanced data: II. Data available on more than two generations. Biometrics 33:497-504. https://doi.org/10.2307/2529364.

Tsuruta, S., I. I. Misztal, D. Lourenco, and T. Lawlor. 2014. Assigning unknown parent groups to reduce bias in genomic evaluations of final score in US Holsteins. J. Dairy Sci. 97:5814-5821. https://doi .org/10.3168/jds.2013-7821.

van Grevenhof, E. M., J. Vandenplas, and M. P. L. Calus. 2019. Genomic prediction for crossbred performance using metafounders. J. Anim. Sci. 97:548-558. https://doi.org/10.1093/jas/sky433.

VanRaden, P. M. 1992. Accounting for inbreeding and crossbreeding in genetic evaluation of large populations. J. Dairy Sci. 75:3136-3144. https://doi.org/10.3168/jds.S0022-0302(92)78077-1.

VanRaden, P. M. 2008. Efficient methods to compute genomic predictions. J. Dairy Sci. 91:4414-4423. https://doi.org/10.3168/jds .2007-0980.

VanRaden, P. M., K. M. Olson, G. R. Wiggans, J. B. Cole, and M. E. Tooker. 2011. Genomic inbreeding and relationships among Holsteins, Jerseys, and Brown Swiss. J. Dairy Sci. 94:5673-5682. https://doi.org/10.3168/jds.2011-4500.

Vitezica, Z. G., I. Aguilar, I. Misztal, and A. Legarra. 2011. Bias in genomic predictions for populations under selection Genet. Res. (Camb.) 93:357-366. https://doi.org/10.1017/ S001667231100022X

Westell, R. A., R. L. Quaas, and L. D. Van Vleck. 1988. Genetic groups in an animal model. J. Dairy Sci. 71:1310-1318. https:// doi.org/10.3168/jds.S0022-0302(88)79688-5.

Wiggans, G. R., T. S. Sonstegard, P. M. VanRaden, L. K. Matukumalli, R. D. Schnabel, J. F. Taylor, F. S. Schenkel, and C. P. Van Tassell. 2009. Selection of single-nucleotide polymorphisms and quality of genotypes used in genomic evaluation of dairy cattle in the United States and Canada. J. Dairy Sci. 92:3431-3436. https: //doi.org/10.3168/jds.2008-1758.

Xiang, T., O. F. Christensen, and A. Legarra. 2017. Genomic evaluation for crossbred performance in a single-step approach with metafounders. J. Anim. Sci. 95:1472-1480. https://doi.org/10 $.2527 /$ jas.2016.1155.

Xu, L., B. Zhu, Z. Wang, L. Xu, Y. Liu, Y. Chen, L. Zhang, X. Gao, H. Gao, S. Zhang, L. Xu, and J. Li. 2019. evaluation of linkage disequilibrium, effective population size and haplotype block structure in Chinese cattle. Animals (Basel) 9:83. https://doi.org/10 $.3390 /$ ani9030083.

\section{ORCIDS}

A. A. Kudinov $\odot$ https://orcid.org/0000-0002-7811-576X

E. A. Mäntysaari @ https://orcid.org/0000-0003-0044-8473

G. P. Aamand (ํ) https://orcid.org/0000-0003-1885-3290

P. Uimari (๑ https://orcid.org/0000-0002-5349-3932

I. Strandén @ https://orcid.org/0000-0003-0161-2618 